\title{
Comparison between uses of topical tacrolimus and triamcinolone ointment for the treatment of oral lichen planus.
}

\author{
Ahmed S ${ }^{1}$, Prodhan M R A², Rezwana R ${ }^{3}$, Afroz J S4, Ahmad M5, Khan M R H6
}

Received: 25.02.18

Accepted: 28.03 .18

Abstract:

Background: Lichen planus is a chronic mucocutaneous disease with primary or secondary involvement of the oral mucosa. In particular in case of erosive lichen planus most of the patients experience no long term symptoms relief of conventional topical treatment such as corticosteroid ointment. Topical tacrolimus applications to mucosal lesions seem to be an alternative treatment approach.

Objective: The aim of this study was to compare the efficacy of topical tacrolimus ointment with that of triamcinolone acetonide ointment on patients suffering from oral lichen planus.

Study design: This was a clinical trial.

Study setting and period: This study was conducted in outpatient department of Oral and Maxillofacial Surgery, Dhaka Dental College in the period between June 2013 to May 2014.

Subjects: A total of sixty patients were enrolled. Out of sixty patients thirty patients used topical tacrolimus $0.03 \%$ (Group A) and another thirty patients used topical triamcinolone acetonide( Group B) for the treatment of oral lichen planus.

Methods: A total of sixty patients were asked to answer a questionnaire regarding the degree of pain and burning sensation in every two weeks according to visual analogue scale (VAS). In every two weeks change of colour and size and also any side effects of these drugs were assessed. Data were analyzed using Statistical Package for Social Science (SPSS), version 15.0 for windows.

Results:At the end of 12th week follow up periods complete relief of pain and burning sensation in group $A$ patients were $100 \%$ and in group B it was $30 \%$. There was no side effect in two study group patients.

1. Dr. Shaikh Ahmed BDS, FCPS (OMS), Assistant Professor, Department of Oral and Maxillofacial Surgery, TMSS Medical College Dental Unit, Bogra

2. Dr. Md. RubayetAlamProdhan BDS, Lecturer, Department of Orthodontic, TMSS Medical College Dental Unit, Bogra

3. Dr. RifatRezwana, BDS, Lecturer, Department of Conservative Dentistry \&Endodontics, TMSS Medical College Dental Unit, Bogra

4. Dr. JahanSadiaAfroz, BDS, BCS (Health), Assistant Dental Surgeon, Gobindagonj Health Complex, Gaibandha.

5. Dr. Mottakin Ahmad, BDS, MPH (NIPSOM), Assistant Professor \& Head, Department of Pediatric Dentistry, TMSS Medical College Dental Unit, Bogra

6. Dr. Md. Rakibul Hassan Khan, BDS, MPH (NIPSOM)Lecturer, Department of Dental Public Health, TMSS Medical College Dental Unit, Bogra.

Correspondence : Dr. Shaikh Ahmed, Mobile: +8801748664414,E-mail: shaikh14cmc@yahoo.com 


\section{Introduction:}

Lichen planus is a chronic autoimmune, mucocutaneous disease. It can affect the oral mucosa, skin, scalp and nails. In the majority of patients with oral lichen planus there is no associated cutaneous lichen planus or lichen planus at other mucosal sites ${ }^{1}$. It commonly affects the middle- aged patients ${ }^{2}$ and has a female predilection. The disease affects $0.5-2 \%$ of the population. The etiology of lichen planus still remains unknown. Lichen planus in believed to result from an abnormal $T$ cell mediated immune response in which basal epithelial cells are recognized as foreign because of changes in the antigenicity of their cell surface ${ }^{3}$. Current data suggest that oral lichen planus is a $T$ cell mediated autoimmune disease in which auto cytotoxic CD8+ T cells trigger the apoptosis of oral epithelial cells ${ }^{4}$. Oral lichen planus was first described clinically by Wilson in 1869 as a chronic mucocutaneous disorder ${ }^{5}$. Cutaneous lichen planus is recurrent, itchy ${ }^{6,7}$ and non-contagious ${ }^{8}$. In most of the patients, oral lichen planus is asymptomatic while some patients may report of a roughness of the lining of the mouth, sensitivity of the oral mucosa to hot or spicy foods, painful oral mucosa, red or white patches on the oral mucosa, or oral ulceration. The clinical history includes phases of remission and exacerbation?. Six clinical forms of oral lichen planus have been described which are white forms namely reticular, papular and plaque- like and red forms namely the erosive(ulcerated), atrophic (erythematous) and bullous $^{10,11}$. Among the types reticular and erosive are the main types ${ }^{12}$. It is not uncommon for the same patient to present with multiple forms of oral lichen planus. Mucosal lesions which are multiple, generally have a symmetrical distribution, particularly on the mucosa of the checks, adjacent to molars, and on the mucosa of the tongue, less frequently on the mucosa of the lips and on the gums (the atrophic and erosive forms located on the gums manifest as a desquamative gingivitis), more rarely on the palate and floor of the mouth ${ }^{8,13}$. A regular follow-up of patients with oral lichen planus should be performed and in suspected cases, biopsy should be provided. Currently the main treatment modalities of oral lichen planus are immunosuppressive drugs since many years, i.e. corticosteroids, are considered as first line of treatment ${ }^{14}$. Other therapeutic modalities for oral lichen planus include general measures (good oral hygiene, antibacterial mouth wash, and anti-fungal agents), intralesional or systemic corticosteroids, topical or systemic retinoids, antimalarial, griseofulvin, dapson, oral PUVA, cyclosporine and surgical techniques (carbon dioxide, ND-YAG lasers, cryotherapy, and excision). Oral lichen planus is frequently resistance to these treatments, thereby it requires trials of new therapeutic modalities ${ }^{15}$. Corticosteroids are the class of drug most commonly used for the treatment of oral lichen planus because of their action in suppressing cell- mediated immune activity ${ }^{16}$. Triamcinolone acetonide paste is the most widely available commercial preparation for the treatment of oral lichen planus. In the race to develop potent alternative immunomodulatory agents, considerable emphasis has been directed toward more selective druds, such as macrolide lactones. One of the lead compounds of this class is tacrolimus which is currently available worldwide for the prevention of organ transplant rejection ${ }^{17,18}$. The safety profile of topical tacrolimus appears to be superior to that of high potency topical steroids when used for chronic dermatomes ${ }^{19}$. Therefore this study was designed to compare the efficacy of topical tacrolimus and topical triamcinolone acetonide in the management of oral lichen.

\section{Materials and methods:}

This clinical trial was conducted during the period June 2013 to May 2014, among two different groups of patients who used topical tacrolimus $0.03 \%$ (Group A) and topical triamcinolone acetonide( Group B) in outpatient department of oral and maxillofacial surgery, Dhaka dental college . After obtaining ethical clearance from the Institutional Ethical Committee, through non probability sampling as convenient to the investigators 60 patients were enrolled who attended the outpatient department of oral and maxillofacial surgery, Dhaka dental college with clinically and histopathologically diagnosed as oral lichen planus. The patient who were suffering from systemic diseases like diabetes mellitus, renal dysfunctions, liver dysfunctions or any other mucosal diseases, skin diseases which may be associated with oral lichen planus, drug therapy which may cause lichen planus like lesion or Histopathological examination with atypical or lichenoid dysplastic feature were excluded from the study. To determine the prevalence and distribution of Lichenplanus among the patients a 
validated research instrument Visual Analogue Scale (VAS) was been used to indicate the severity of pain following application of topical agents in oral lichen planus of selected patients. Data were analyzed using Statistical Package for Social Science (SPSS), version 15.0 for windows to determine frequency, distributions, means and proportions. Comparison of proportions was done by using Chi square test, $p$ value of less than 0.001 was considered to be statistically significant.

\section{Results:}

Total 60 patients were evaluated for the comparison of the effect between uses of topical tacrolimus and topical triamcinolone for the treatment of oral lichen planus. Out of 60 patients, 30 patients was applied topical tacrolimus $0.03 \%$ that was group $A$ and 30 were topical triamcinolone acetonide that was group $B$. Age range of patients were 26 year to 60 year in group $A$ and 22 year to 59 year in group $B$.

The findings obtained from data analysis are presented here.

Table 1: Age distribution in two study groups $(n=60)$

\begin{tabular}{|c|c|c|c|}
\hline Age & $\begin{array}{l}\text { Group A } \\
\text { (Tarcolimus) } \\
(\mathrm{n}=30) \\
\text { No (\%) }\end{array}$ & $\begin{array}{c}\text { Group B } \\
\text { (Triamcinolone) } \\
\begin{array}{c}(\mathrm{n}=-60) \\
\text { No }(\%)\end{array}\end{array}$ & $\begin{array}{c}\mathrm{p} \\
\text { value }\end{array}$ \\
\hline $\begin{array}{c}20-30 \\
\text { yrs }\end{array}$ & $2(6.7 \%)$ & $7(23.3 \%)$ & \\
\hline $\begin{array}{c}31-40 \\
\text { yrs }\end{array}$ & $6(20.0 \%)$ & $10(33.3 \%)$ & \\
\hline $\begin{array}{l}41-50 \\
\text { yrs }\end{array}$ & $19(63.3 \%)$ & $11(36.7 \%)$ & \\
\hline$>50 \mathrm{yrs}$ & $3(10.0 \%)$ & $2(6.7 \%)$ & \\
\hline Total & $30(100.0 \%)$ & $30(100.0 \%)$ & \\
\hline $\begin{array}{c}\text { MeantSD } \\
\text { Range }\end{array}$ & $\begin{array}{c}44.0 \pm 7.75 \\
26-60\end{array}$ & $\begin{array}{c}37.67 \pm 9.73 \\
22-59\end{array}$ & $0.007^{2}$ \\
\hline
\end{tabular}

Table 1 demonstrates highest percentage (63.3\%) in group A and highest percentage (36.7\%) in group B were from the age group 41-50 years.

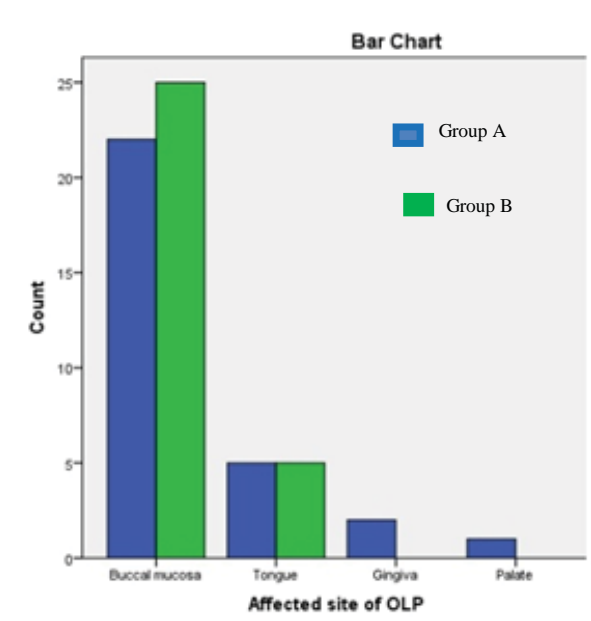

Figure 1:demonstrates highest percentage of affected site of OLP was $73.3 \%$ in group A and $83.3 \%$ in group $B$ patients in buccal mucosa.

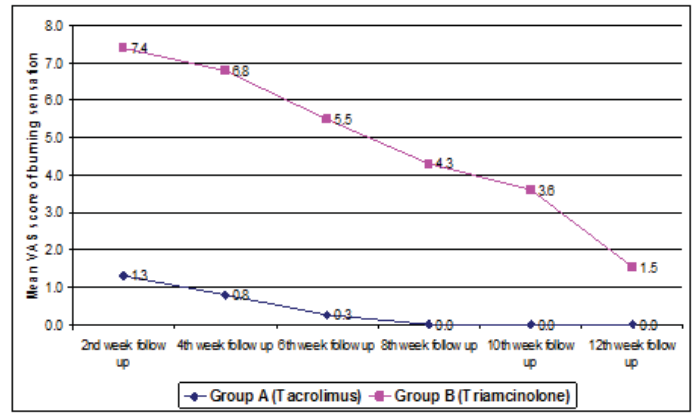

Figure 2: Line graphs showing mean VAS score of pain in two study group.

The line graphs shows mean VAS of pain after 2nd week of follow up periods in Group - A patients was 1.6 which was reduced to 0 after 12 th week of follow up periods. In group B patients mean VAS was 7.9 after 2nd week follow up periods which was reduced to 2.3 after 12 th week follow up periods. Vas score 1.6 refers to mild pain and 0 no pain. As score 7.9 refers to severe pain and 2.3 mild pain.

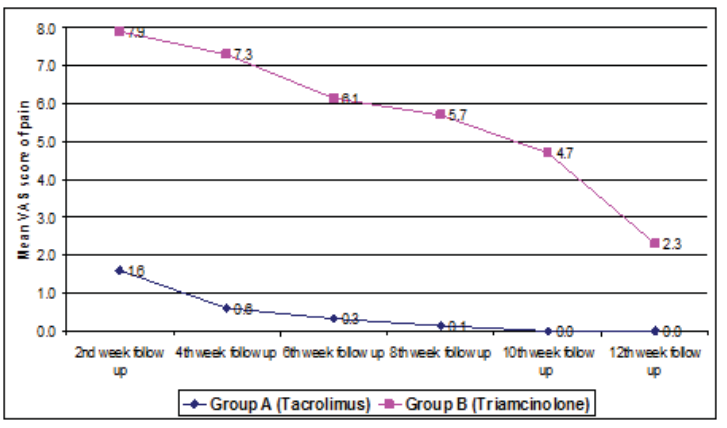

Figure 3: Line graph shows mean VAS of burning 
sensation in two study groups.

Above line graphs shows mean vas score of burning was 1.3 after 2 nd week of follow up periods in group A patients which was reduced to 0 after 12th week of follow up periods. In group B patients mean vas score of burning was 7.4 after 2 nd week of follow up periods which was reduced to 1.5 after 12 th week of follow up periods. As score 1.3 refers to mild burning and 0 no burning. Vas score 7.4 refers to severe burning and 1.5 mild burning.

\section{Discussion:}

This comparative study was carried out in the department of oral and maxillofacial surgery Dhaka dental College from June 2013 to May 2014 with a sample size of 60 patients in two groups. In group A 30 patients applied topical tacrolimus and in group B 30 patients applied topical triamcinolone acetonide for the treatment of oral lichen planus. Lichen planus is a chronic inflammatory mucocutaneous disease that occurs in about 0.02 to $4 \%$ of general population affecting skin and / or mucosa. The treatment of symptomatic oral lichen planus represents a perplexing therapeutic challenge. Multiple topical and systemic treatments for oral lichen planus have been reported to be effective including topical and systemic corticosteroids, griseofulvin, hydroxychloquine, dapson, topical retinoids, and topical cyclosporine. Topical tacrolimus has been reported to be effective treatment for oral lichen planus, including those forms that has been recalcitrant to treatment. Patients attending the outpatient department of oral and maxillofacial surgery with clinically and histopathologically diagnosed as an oral lichen planus were included in this study.In group A patients topical tacrolimus $0.03 \%$ and in group B patients topical triamcinolone were applied 2-3 times daily for 3 months and follow up were given in every 2 weeks to assesses symptoms relief like pain and burning sensation, change of color, change of size and also side effect of burning of these drugs. Age and sex of patients have not played any role to comparison between uses of topical tacrolimus and topical triamcinolone for the treatment of oral lichen planus. In this study maximum result $50 \%$ (30) patients were within 41-50 years age group and58.3\% (34) of patients were female. This study lesion result is consistent with some other study20. In this study oral lichen planus commonly affects buccal mucosa. Out of 60 patients 47 patients $(78.3 \%)$ had lesion in buccal mucosa that was adjacent to gum. Involvement of tongue was in 10 patients, gingiva 2 patients, and palate 1 patient. This study is assimilar as other study that is mucosal lesions which are multiple, particularly on the mucosa of check, adjacent to molars, and on the mucosa of the tongue, less frequently on the mucosa of the lips and on the gums, more rarely on the palate and floor of the mouth ${ }^{8,13}$. In this study predominant form of oral lichen planus was erosive or ulcerative pattern. Out of 60 patients 58 $(96.6 \%)$ patients were erosive form. This study is consistent with other study that is ; six clinical forms of oral lichen planus have been described which are white forms namely reticular, papular, plaque like and red form namely the erosive (ulcerative), atrophic (erythematous) and bullous. Among all the types, reticular and erosive are the main type ${ }^{12}$. At the end of 2nd week follow up, pain score (vas score) in group A patients were complete relief of pain in $12(40 \%)$ patients and no complete relief of pain in group B patients. Complete burning sensation relief was in $21(70 \%)$ patients in group A and no relief of burning sensation in group $B$ patients. Change of colour and size were $24(80 \%)$ and $23(76.7 \%)$ in group A patients whereas no change of colour and size in group $B$ patients. Burning sensation as a side effect in $9(30 \%)$ patients in group A and no side effect in group $B$ patients. This study at the end of 4 th week follow up, pain score (vas score) in group A patients were complete relief of pain in $23(76.3 \%)$ of patients and moderate relief of pain in $10(33.3 \%)$ patients in group B. Complete burning sensation relief was in 24 $(80 \%)$ patients in group A and moderate relief of burning in $15(50 \%)$ patients in group $B$. Change of colour and size in group A patient.In where, 28 $(93.3 \%)$ and $25(83.3 \%)$ whereas change of size was $1(3.3 \%)$ patients in group B. This study is as similar as other study ${ }^{21}$. At the end of 6th week follow up, complete relief of pain were $25(83.3 \%)$ patients in group A and moderate relief of pain were 22 (73.3\%) patients in group B. Complete burning relief were 26 $(86.7 \%)$ patients in group A and moderate relief of burning were $22(73.3 \%)$ patients in group $B$ patients. Change of colour and size were $30(100 \%)$ and 29 (96.7\%) patients of group A whereas it was 4 (13.3\%) and $3(10 \%)$ patients of group B. No side effects were found in two study group patients. In this study at the end of 8th week follow up, complete relief of pain in group A patients were 28 ( $93.3 \%$ ) and moderate pain relief in 28 ( 93.3\%) patients of group B. Complete burning relief were $30(100 \%)$ patients in group $A$ and mild burning were $13(43.3 \%)$ patients in group $B$. Change of colour and size in group A were $100 \%$ and in group $B$ it was $40 \%$ and $36.7 \%$. At the end of 10 th week follow up, complete relief of pain in group $A$ 
were $30(100 \%)$ patients and mild pain were in 10 $(33.3 \%)$ patients in group B. Burning relief were 30 $(100 \%)$ patients in group $A$ and mild burning in 15 $(30 \%)$ patients in group B. Changes of colour and size in group A were $30(100 \%)$ patients whereas it was $18(60 \%)$ patients in group B. In this study at the end of 12th week follow up, complete relief of pain and burning sensation (vas score) in group A were 30 $(100 \%)$ patients and complete relief of pain and burning sensation were $4(13.3 \%)$ and $9(30 \%)$ patients in group B. Mild pain and burning were $22(73.3 \%)$ and $20(66.7 \%)$ patients in group B. Change of colour and size in group A were $30(100 \%)$ patients whereas it was $24(80 \%)$ and 25 ( $83.3 \%)$ patients in group B. There is no side effect in two study group patients. This study result is as similar as other study ${ }^{22}$.

\section{Conclusion:}

On the basis of the clinical examination and the statistical analysis of collected data, the following conclusions could be drown. Topical tacrolimus appears to be effective in the management of oral lichen planus as it produce statistically significant in both the signs and symptoms of the disease, with $100 \%$ of subject showing complete cure. No significant adverse effectswere noticed in any of the subjects. Burning sensation were noticed in 9 patients following first and second week of treatment. Triamcinolone acetonide also produce significant relief from the symptoms.

\section{Reference:}

1) Al-Hashimi I, Schifter M, Lockhart PB, Wray D, Brennan M, Migliorati CA, et al. Oral lichen planus and oral lichenoid lesions: diagnostic and therapeutic consideration. Oral Surg Oral Pathol Oral RadiolEndod 2007; 103 suppl: S25. El-12.

2) Sugerman PB, Savage NW, Walsh LJ, Zhao ZZ, Zhok $\mathrm{XJ}$, Khan A, et al. The pathogenesis of oral lichen planus.Crit Rev Oral Biol Med 2002; 13:350-65.

3) Sapp JP, Eversole LR, Wysocki GP. Contemporary oral and maxillofacial pathology. St Louis(MI): Mosby; 1997.

4) Sumairi B, Ismail, Satish K.S. Kumar, Rosnah B. Zain. Oral lichen planus and lichenoidreaction: etiopathogenesis, diagnosis, management and malignant transformation. Journal of Oral Science, vol. 49, no.2, 89-lk106,2007.

5) Wilson E. On lichen planus.J cutan Med Dis Skin 1869; 3: 117-132.

6) KattaR.Lichenplanus.AmFam physician 2000; 61: 3319-24, 3327-8.

7) Sapuppo A, Lanza G. Lichen ruberplanus In: Lanza G, ed. AnatomiaPathologicaSistematica, vol. 1, 2nd ed. Padova:Piccin1985:974-5.

8) LeighebG.Testo e Atlante di Dermatologia. Pavia: Edizio- ni Medico Scientifiche; 1995: 207-13.

9) Eisen D.The evaluation of cutaneous, genetal, scalp, nail, esophageal, and ocular involvement in patients with oral lichen planus.Oral Surg Oral Med Oral Pathol Oral RadiolEndod 1999;88431-6.

10) Andreasen JO. Oral lichen planus.I.A clinical evaluation of 115 cases. Oral Surg Oral Med Oral Pathol 1968; 25:3142.

11) Pindborg JJ, Reicharf PA, Smith CJ, Vander Waal I (1997) Histological typing of cancer and precancer of oral mucosa. 2nded,Springer, New York,30.

12) Mollaoglu N. Oral lichen planus: A review. Bri J Oral Maxillofacial Surg 200; 38(4): 370-7.

13) Laskaris G. Colar Atlas of Oral Disease. Stuttgart: Georg thiemeVerlag: 1994.

14) Ismail Sumairi b, Kumar satish KS, ZainRosnah B. Oral lichen planus and lichenoid reactions: Etiopathogenesis, Diagnosis, management and malignant transformation. Journal of Oral Science 2007; 49(2):89-106.

15) RozyckiTW, RogersRS, Pittelkow MR, Marian TM. Topical tacrolimus in the treatment of symptomatic oral lichen planus: A series of 13 patients. J AM AcadDermatol 2002; 46: 27-34.

16) Ismail Sumairi b, Kumar satish KS, ZainRosnah B. Oral lichen planus and lichenoid reactions: Etiopathogenesis, Diagnosis, management and malignant transformation. Journal of Oral Science 2007; 49(2):89-106.

17) Ruzicka T, Assmann T, Tacrolimus Homey B. The drug for the turn of millennium.Arch Dermat 1999; 135:574-80.

18)Lozada-NurFrancinal,SroussiHerve Y. Tacrolimus Powder in orabase $0.1 \%$ for the treatment of oral lichen planus and lichenoid lesions: An open clinical trial. Oral Surg Oral Med Oral Pathol Oral RadiolEndod 2004; 98:673-78.

19) Antonio Bulbena, GuillemPhailhez, JoaquimColl, Richard Balon. Attitudes and views on psychiatry: a comparison between Spanish and US medical students. Acad Psychiatry 2005; 29(1): 82-91.

20) Swarna YM, Ali IM, Rajeshwari G Anigeri, Devarasa GM. A comparative evaluation of efficacy of tacrolimus and triamcinolone acetonide in the management of symptomatic oral lichen planus. Journal of indian Academy of oral medicine and radiology,July-September 2011; 23(3): 184189.

21) A. Eckardta, B. Volkerb, O.Starkea, Y.Kaeverc, A. Kappb. Topical tacrolimus in erosive oral lichen planus: An effective treatment approach. Oral Biosci Med 2005; 4:235-240.

22) Javed A Qazi. Treatment of oral lichen planus with topical ointment. Pakistan Oral and Dental Journal june2010; vol 30(1): 19-2 